\title{
A prospective, controlled study on the utility of rotational thromboelastometry in surgery for acute type A aortic dissection
}

\author{
Mårten Larsson ${ }^{1}$, Igor Zindovic ${ }^{1}$, Johan Sjögren ${ }^{1}$, Peter Svensson J², Karin Strandberg ${ }^{3}$, \\ and Shahab Nozohoor ${ }^{1}$ \\ ${ }^{1}$ Lund University Skåne University Hospital Department of Clinical Sciences Lund \\ Department of Cardiothoracic Surgery Lund Sweden \\ ${ }^{2}$ Department of Coagulation Disorders Skåne University Hospital Lund University \\ Malmö Sweden \\ ${ }^{3}$ Department of Clinical Chemistry Malmö Sweden
}

March 7, 2022

\begin{abstract}
Objective: To evaluate the hemostatic system with ROTEM in patients undergoing surgery for acute type aortic dissection (ATAAD) using elective aortic procedures as controls. Design: This was a prospective, controlled, observational study. Setting: The study was performed at a tertiary referral center and university hospital. Participants: Twenty-three patients with ATAAD were compared to 20 control patients undergoing elective surgery of the ascending aorta or the aortic root. Results: ROTEM (INTEM, EXTEM, HEPTEM and FIBTEM) was tested at 6 points in time before, during and after surgery for ATAAD or elective aortic surgery. The ATAAD group had an activated coagulation coming into the surgical theatre. The two groups showed activation of both major coagulation pathways during surgery, but the ATAAD group consistently had larger deficiencies. Reversal of the coagulopathy was successful, although none of the groups reached elective baseline until postoperative day 1. ROTEM did not detect low levels of clotting factors at heparin reversal nor low levels of platelets. Conclusions: This study demonstrated that ATAAD is associated with a coagulopathic state. Surgery causes additional damage to the hemostatic system in ATAAD patients as well as in patients undergoing elective surgery of the ascending aorta or the aortic root. ROTEM does not adequately catch the full coagulopathy in ATAAD. A transfusion protocol in ATAAD should be specifically created to target this complex coagulopathic state and ROTEM does not negate the need for routine laboratory tests.
\end{abstract}

\section{A prospective, controlled study on the utility of rotational thromboelastometry in surgery for acute type A aortic dissection}

Mårten Larsson $\mathrm{MD}^{1}$, Igor Zindovic, MD $\mathrm{PhD}^{1}$, Johan Sjögren $\mathrm{MD} \mathrm{PhD}^{1}$, Peter J Svensson MD $\mathrm{PhD}^{2}$, Karin Strandberg MD $\mathrm{PhD}^{3}$, Shahab Nozohoor MD $\mathrm{PhD}^{1}$

${ }^{1}$ Lund University, Skåne University Hospital, Department of Clinical Sciences, Lund, Department of Cardiothoracic Surgery, Lund, Sweden; ${ }^{2}$ Department of Coagulation Disorders, Skåne University Hospital, Lund University, Malmö, Sweden; ${ }^{3}$ Department of Clinical Chemistry, Malmö, Sweden

Corresponding author: Mårten Larsson, MD

Department of Cardiothoracic Surgery

Skåne University Hospital, SE-221 85 Lund, Sweden

Phone: +46 46-171000; E-mail: marten.larsson@med.lu.se 
Funding: This research did not receive any specific grant from funding agencies in the public, commercial, or not-for-profit sectors.

Conflict of interest: None to declare

Data availability: Not possible due to ethics approval

This study was approved by the Ethics Committee for Clinical Research at Lund University, Sweden (ref. 2015/197)

Patient consent: Before inclusion a consent was gathered from the patient or the next of kin (oral and/or written)

\section{ABSTRACT}

Objective: To evaluate the hemostatic system with ROTEM in patients undergoing surgery for acute type aortic dissection (ATAAD) using elective aortic procedures as controls.

Design: This was a prospective, controlled, observational study.

Setting: The study was performed at a tertiary referral center and university hospital.

Participants: Twenty-three patients with ATAAD were compared to 20 control patients undergoing elective surgery of the ascending aorta or the aortic root.

\section{Results:}

ROTEM (INTEM, EXTEM, HEPTEM and FIBTEM) was tested at 6 points in time before, during and after surgery for ATAAD or elective aortic surgery. The ATAAD group had an activated coagulation coming into the surgical theatre. The two groups showed activation of both major coagulation pathways during surgery, but the ATAAD group consistently had larger deficiencies. Reversal of the coagulopathy was successful, although none of the groups reached elective baseline until postoperative day 1. ROTEM did not detect low levels of clotting factors at heparin reversal nor low levels of platelets.

Conclusions: This study demonstrated that ATAAD is associated with a coagulopathic state. Surgery causes additional damage to the hemostatic system in ATAAD patients as well as in patients undergoing elective surgery of the ascending aorta or the aortic root. ROTEM does not adequately catch the full coagulopathy in ATAAD. A transfusion protocol in ATAAD should be specifically created to target this complex coagulopathic state and ROTEM does not negate the need for routine laboratory tests.

KEY WORDS: Aorta, Aneurysm, Dissection, Bleeding, Rotational thromboelastometry, ROTEM

\section{INTRODUCTION}

Acute type A aortic dissection (ATAAD) is a lethal condition requiring emergent aortic surgery. The procedure is challenging and associated with high rates of peri-operative bleeding and blood product transfusions. Mortality is high with rates ranging from $16-18 \%$ after surgery in large registries ${ }^{1-3}$. A significant cause of the high mortality and morbidity is peri-operative bleeding ${ }^{4}$. The impairment of the coagulation system is caused by the contact of blood with the non-endothelialized walls of the false lumen, but other triggers also have been suggested ${ }^{5-7}$. Surgery for acute type A aortic dissection is conducted using a cardiopulmonary bypass circuit and usually a deep hypothermic state, both of which further increase the coagulopathy.

Transfusion strategy in major aortic surgery may either be reactive, with the administration of blood products in response to the development of a clinical coagulopathy, or pre-emptive, based on the administration of blood products to prevent a clinically detectable coagulopathy. Both approaches lead to a significant use of blood products. A recent review of bleeding management in vascular surgery concluded that hemostasis should be monitored, and goal directed ${ }^{8}$. This calls for alternative bleeding management, and the adoption of a transfusion algorithm guided by rotational thromboelastometry (ROTEM) could be an option. 
At many centers, management of perioperative bleeding is carried out according to rotational ROTEMguided protocol for transfusion of blood products and procoagulants, reducing the need for transfusions and decreasing the risk of bleeding after cardiac surgery ${ }^{9-12}$.

A prospective study by Ogawa et al. ${ }^{13}$ compared values obtained using standard laboratory coagulation tests (PT(INR), APTT and fibrinogen) with ROTEM CT and MCF values for the parameters INTEM, EXTEM, HEPTEM, and FIBTEM in adult patients undergoing cardiac surgery and demonstrated that some ROTEM measurements could act as surrogates for standard coagulation tests. However, although reference ROTEM values in patients undergoing elective and non-complex cardiac surgery have been described ${ }^{14-16}$, similar data are lacking in the acute and complex setting. Furthermore, few studies have investigated the dynamics of the key components of the coagulation system visualized by ROTEM in association with the complex coagulopathic setting of ATAAD surgery. It is therefore unclear whether the ROTEM-guided treatment algorithm utilized in routine surgery also may be used when performing ATAAD repair.

To assess the performance of ROTEM during surgery for ATAAD, we reviewed ROTEM in surgically treated patients with ATAAD compared to a control group undergoing elective aortic surgery.

\section{METHODS}

This study was approved by the Ethics Committee for Clinical Research at Lund University, Sweden (ref. $2015 / 197)$. As this was one of several studies derived from the same prospective project, the study population and methods have been described previously ${ }^{17}$.

\section{Study population}

A total of 27 patients with ATAAD were selected for the study. Of those, four patients were excluded: two because they did not follow surgical protocol, one because of intraoperative death, and one because of a lack of resources. The control group consisted of 20 patients who underwent elective surgery of the ascending aorta and/or the aortic root. A preoperative written or oral consent by the patient or the next of kin was retrieved before any collection of data in all ATAAD patients. A preoperative written consent was obtained by all elective control patients.

\section{Study Design}

This was a prospective, single center, observational study comparing patients undergoing surgery for ATAAD with a control group consisting of patients undergoing elective aortic surgery of the ascending aorta and/or the aortic root. The ATAAD group consisted of patients over the age of 18 with symptom duration $<48 \mathrm{~h}$ undergoing surgery for ATAAD at Skåne University Hospital, Lund, Sweden, between September 2015 and April 2018. ATAAD was confirmed by contrast-enhanced computed tomography. The anatomical extent of the dissection was defined according to the Stanford ${ }^{18}$ and Debakey ${ }^{19}$ classification. Exclusion criteria were preoperative use of anti-coagulants or anti-platelet drugs other than aspirin (both groups) and if surgical approach deviated from routine (as described below) (ATAAD group).

All patients with acute aortic syndromes (e.g. ATAAD and intramural hematomas) referred to our clinic during the study period were registered and screened for inclusion (Fig 1). Routinely, a ROTEM Delta (Tem Innovations GmgH, Germany) and standard lab test guided bleeding management protocol was used at our clinic (Fig 2). Red blood cell transfusions were given at B-Hemoglobin $<90 \mathrm{~g} / \mathrm{L}$. Platelets were administered at maximum clot firmness (MCF) EXTEM $<50 \mathrm{~mm}$ and MCF FIBTEM $>10 \mathrm{~mm}$ or platelet count $<100 \times 10^{9} / \mathrm{L}$. Fibrinogen and/or plasma were used at MCF FIBTEM $<15 \mathrm{~mm}$ or P-fibrinogen $<2 \mathrm{~g} / \mathrm{l}$. Plasma or prothrombin complex concentrate $(\mathrm{PCC})$ were used at coagulation time $(\mathrm{CT})$ EXTEM $>100 \mathrm{~s}$, CT INTEM $>240 \mathrm{~s}$, P-PT(INR) $>1.5$ or P-APTT $>1.5 \times$ normal value. Additional Tranexamic acid was used when maximum lysis (ML) exceeded 15\%. However, final decision regarding transfusions was at the surgeon's discretion.

\section{Endpoints}


The primary endpoints were measurements of ROTEM values of clotting time (CT) in INTEM, EXTEM, and HEPTEM and maximum clot firmness (MCF) in EXTEM and FIBTEM. Secondary endpoints were need for transfusion with blood products, procoagulants used, 30-day mortality, bleeding at $24 \mathrm{~h}$, and reoperation for bleeding.

\section{Definitions}

An acute type A aortic dissection was defined as a separation of the aortic layers providing a true and a false lumen for the blood flow, presenting within 14 days of symptom onset and extending distally from the aortic root or ascending aorta (Stanford A). Surgical mortality was defined as death within 30 days of surgery. Intraoperative bleeding was defined as blood loss collected and quantified using intraoperative cell salvage and surgical gauze swabs. Postoperative stroke was defined as a permanent loss of neurological function caused by an ischemic event with or without confirmation with computed tomography or magnetic resonance imaging. Renal replacement therapy (RRT) was defined as any need for continuous hemofiltration or hemodialysis postoperatively. Cardiac tamponade was defined as pericardial effusion that required pericardial drainage or re-exploration.

\section{Biomarker Measurements}

Samples of blood were collected at six points in time: $T_{0}$ - anesthesia induction; $T_{1}$ - lowest core temperature (only collected in elective patients with core temperature [?]32degC, $\mathrm{n}=14$ ); $\mathrm{T}_{2^{-}}$just before protamine administration; $\mathrm{T}_{3}$ - end of surgery; $\mathrm{T}_{4^{-}} 24 \mathrm{~h}$ after surgery; $\mathrm{T}_{5^{-}}$-96-120h after surgery. A central venous line was used for samples collected at $\mathrm{T}_{0}, \mathrm{~T}_{4}$, and $\mathrm{T}_{5}$. A circuit from the cardiopulmonary bypass machine was used for samples collected at $T_{1}$ and $T_{2}$. A radial artery line was used for the samples collected at $T_{3}$.

All samples were analyzed at the Dept. of Clinical Chemistry, Division of Laboratory Medicine, Skane University Hospital, Lund, Sweden, on a ROTEM. Parameters tested were INTEM, EXTEM, HEPTEM, and FIBTEM and variables clotting time (CT), which is time from start until clotting is initiated in seconds (s) and maximum clot firmness (MCF), which is maximum diameter of clot in millimeters $(\mathrm{mm})$.

\section{Surgical Procedures}

The surgical technique for ATAAD used at our center has been described previously ${ }^{17}$. Median sternotomy, cardiopulmonary bypass (CPB) with hypothermic circulatory arrest and cold blood cardioplegia was used in all cases. Heparin was reversed with protamine after weaning from $\mathrm{CPB}$ and transfusion of procoagulants and blood products initiated. The cooling strategy used in the elective controls were hemiarch procedure $25 \mathrm{degC}$; valve sparing root replacement (ad modum David): 30degC; root replacement (ad modum Bentall) or aortic valve replacement with supracoronary replacement of the ascending aorta: $32 \mathrm{degC}$; isolated replacement of the ascending aorta: $32-36 \operatorname{deg}$.

During the study period, all patients received preoperative tranexamic acid (2-3g preoperatively and 1-2g after termination of CPB for a total of $4 \mathrm{~g}$ ) to prevent hyperfibrinolysis. A Dideco Electa (Sorin Group, Electa, Italy) cell saver machine was used to process and re-infuse salvaged blood. Heparin dose was calculated using the Hepcon HMS Plus system (Medtronic, Minneapolis, MN, USA) to reach an activated clotting time $(\mathrm{ACT})$ of $>480 \mathrm{~s}$ and monitored serially during $\mathrm{CPB}$ to maintain ACT $>480$ s with additional heparin added if necessary. Antithrombin was administered if the heparin dose response slope was low $(<70 \mathrm{~s} / \mathrm{U} / \mathrm{ml})$ and a subsequent test was conducted ten minutes later. Two patients in each group received 2000IE of antithrombin during surgery. ACT was routinely controlled at the end of surgery, and additional protamine was given if ACT exceeded 120 seconds.

\section{Statistical Analysis}

Categorical variables were expressed as numbers and percentages. Continuous variables were expressed as mean (+-SD) when normally distributed, otherwise as median [IQR]. ROTEM variables were visualized using box plots. ROTEM reference values are illustrated as dashed lines and shaded areas and are based on reference ranges by Lang et al ${ }^{20}$. Proportions were compared using the chi-square test. Fisher's exact 
test was used when the number of cases was less than 5 , and continuous variables were evaluated using the Mann-Whitney test. Wilcoxon signed-rank test was used for analyzing related samples. Missing values were not analyzed. A $p$-value $<0.05$ was considered statistically significant and analyses were performed using standard software (IBM Corp. Released 2019. IBM SPSS Statistics, Version 26. Armonk, NY: IBM Corp).

\section{RESULTS}

\section{Baseline and Intraoperative Data}

Patient characteristics are presented in Table 1. Bicuspid aortic valve was less common in the ATAAD group ( $0 \%$ vs $32 \%, p<0.01)$, and proximal surgery was less extensive with fewer root replacements in the ATAAD group $(p<0.001)$ compared to the control group. Cardiopulmonary bypass time was shorter in the ATAAD group $(160(+-36)$ min vs $208(+-60)$ min, $p<0.01)$ as well as aortic cross-clamping (75 (+-31) min vs 139 $(+-44) \min , p<0.001)$. The lowest core temperature was significantly lower in the ATAAD group (20.0 $(+-2.8) \operatorname{deg} \mathrm{C}$ vs $30.5(+-3.1) \operatorname{deg} \mathrm{C}, p<0.001)$.

\section{Biomarker Measurements}

ROTEM data is presented in Figure 3. At $\mathrm{T}_{0}$ there was no significant difference in clotting time (CT) in INTEM or HEPTEM between groups (159s [140-170] vs 170s [159-180], $p=0.08$ and 156s [138-168] vs 163s [150-180], $p=0.21)$. During CPB, HEPTEM CT increased markedly in both groups and was significantly higher in the ATAAD group at $\mathrm{T}_{2}(212 \mathrm{~s}[202-239]$ vs $198 \mathrm{~s}$ [193-236], $p=0.05)$. At the end of surgery $\left(\mathrm{T}_{3}\right)$, the HEPTEM CT recovered, although not to preoperative levels and was significantly higher in the ATAAD group (187s [166-203] vs 166s [151-173], $p<0.01$ ). The CT in INTEM and HEPTEM was significantly more prolonged in the ATAAD group at $\mathrm{T}_{3}$ compared to $\mathrm{T}_{0}$ (191s [179-211] vs $159 \mathrm{~s}$ [140-170], $p<0.01$ and $187 \mathrm{~s}$ [166-203] vs 156s [138-168], $p<0.01)$. In the control group, INTEM CT was significantly longer at $\mathrm{T}_{3}$ compared to $\mathrm{T}_{0}(184 \mathrm{~s}$ [162-196] vs $170 \mathrm{~s}$ [159-180], $p=0.03)$ but not HEPTEM CT (166s [151-173] vs 163s [150-180], $p=0.93)$.

\section{EXTEM}

There was a significant difference in EXTEM CT at $\mathrm{T}_{2}(87 \mathrm{~s}$ [76-107] vs 76s [73-84], $p=0.03)$ and a trend towards it at $\mathrm{T}_{3}(59 \mathrm{~s}[54-67]$ vs $64 \mathrm{~s}[60-68], p=0.07)$. At the end of surgery, CT in the control group was significantly longer than preoperative levels (64s [60-68] vs 60s [56-65], $p=0.05)$ and in the ATAAD group there was a trend towards shorter CT compared to preoperative (59s [54-67] vs 62s [56-71], $p=0.06$ ).

The maximum clot firmness (MCF) in EXTEM was lower in the ATAAD group compared to the control group at $\mathrm{T}_{1}, \mathrm{~T}_{2}$, and $\mathrm{T}_{5}(41 \mathrm{~mm}$ [34-41] vs $55 \mathrm{~mm}$ [51-61],p<0.001, 50mm [44-55] vs $57 \mathrm{~mm}$ [54-62],p=0.001 and $69 \mathrm{~mm}[66-72]$ vs $73 \mathrm{~mm}[72-74], p=<0.01$, respectively) and had a similar trend at $\mathrm{T}_{0}(59 \mathrm{~mm}$ [56-65] vs $64 \mathrm{~mm}[58-68], p=0.07)$. EXTEM MCF was also lower in the control group at the end of surgery compared to preoperative levels $(61 \mathrm{~mm}$ [59-65] vs $64 \mathrm{~mm}$ [58-68], $p=0.02)$ but not in the ATAAD group (61mm [58-64] vs $59 \mathrm{~mm}[56-65], p=0.15)$.

\section{FIBTEM}

The preoperative FIBTEM MCF was significantly lower in the ATAAD group compared to the control group (12.0mm [10.0-15.0] vs $16.5 \mathrm{~mm}[13.5-20.0], p=0.03)$, and the pattern persisted during the operation $\left(\right.$ at $\mathrm{T}_{1}$ $9.0 \mathrm{~mm}[5.5-11.5]$ vs $13.0 \mathrm{~mm}[10.0-16.3], p<0.01$ and at $\mathrm{T}_{2} 9.0 \mathrm{~mm}[7.0-12.0]$ vs $13.5 \mathrm{~mm}$ [11.3-15.0], $\left.p<0.01\right)$. There was no difference in FIBTEM MCF at the end of surgery $\left(\mathrm{T}_{3}\right)$ or the first postoperative day $\left(\mathrm{T}_{4}\right)$ $\left(p=0.91\right.$ and $p=0.51$, respectively), however at $\mathrm{T}_{5}, \mathrm{MCF}$ was again lower in the ATAAD group (28.5mm [25.0-37.3] vs 37.0mm [35.0-38.0], $p<0.01)$.

\section{Bleeding, Transfusions and Medical Management}

Data presented in Table 2 reveal that patients in the ATAAD group received significantly more transfusions and procoagulants during surgery and the first 24 hours after surgery. Transfusion of packed red blood cells $(3.3 \mathrm{U}(+-4.0)$ vs $1.2 \mathrm{U}(+-1.5), p=0.03)$, platelets $(4.3 \mathrm{U}(+-1.7)$ vs $2.5 \mathrm{U}(+-1.6), p=0.001)$ and FFP $(2.4 \mathrm{U}$ 
$(+-2.6)$ vs $0.6 \mathrm{U}(+-1.2), p<0.01)$ was larger in the ATAAD group. The use of fibrinogen $(6.0 \mathrm{~g}(+-3.4)$ vs $3.1 \mathrm{~g}(+-2.5), p<0.01)$, PCC $(1780 \mathrm{IU}(+-1050)$ vs $775 \mathrm{IU}(+-896), p<0.01)$ and recombinant factor VIIa $(5(22 \%)$ vs $0(0 \%)$ patients, $p=0.03)$ also were significantly larger in the ATAAD group. No patient was reoperated for bleeding in both groups and postoperative bleeding volumes at $12 \mathrm{~h}(492 \mathrm{ml}(+-235)$ vs $498 \mathrm{ml}$ $(+-175), p=0.64)$ and $24 \mathrm{~h}(810 \mathrm{ml}(+-400)$ vs $740 \mathrm{ml}(+-215), p=0.78)$ did not show any difference between groups.

\section{DISCUSSION}

The results of this study demonstrate that there are predictable and quantifiable changes in ROTEM values during surgery in ATAAD and elective aortic surgery with CPB. Surgery significantly and negatively impacted all ROTEM values assessed in this study (EXTEM CT, INTEM CT, HEPTEM CT, EXTEM $\mathrm{MCF}$, and FIBTEM MCF). The greatest impairment in coagulation parameters occurred consistently in patients with ATAAD. This study demonstrated that ATAAD caused an activation of the coagulation shown in ROTEM prior to surgery $\left(T_{0}\right)$, which developed to a coagulopathy during CPB $\left(T_{1}\right.$ and $\left.T_{2}\right)$ and was not fully recovered compared to elective controls at wound closure $\left(\mathrm{T}_{3}\right)$ despite significantly greater use of procoagulants and transfusions. Our ROTEM-guided transfusion protocol does not seem to catch the full need of transfusions as most of our patients received more transfusions than what ROTEM would suggest. However, bleeding volumes and the need for re-exploration for bleeding or tamponade did not differ and had favorable outcomes in both groups.

Aortic dissection leads to blood being exposed to tissue factor, extracellular collagen, and other subendothelial structures that activate the coagulation process. This is evident by the decreased MCF in FIBTEM indicating consumption of fibrinogen. MCF in EXTEM also was decreased but did not reach the level of significance. Both EXTEM and INTEM CT showed a trend towards longer clotting time indicating reduced amounts of clotting factors. Combined, this indicates an established activation of coagulation when ATAAD develops resulting in a consumption coagulopathy. During surgery, both groups showed similar trends in all ROTEM variables, but the ATAAD group had consistently more impaired values, which could indicate that the dissection consumed coagulation factors, platelets, and fibrinogen prompted by more profound hypothermia.

At the end of surgery, both groups showed similar ROTEM findings. The ATAAD group had longer HEPTEM CT than the control group, but not INTEM CT. This indicates a reduction of factors in the intrinsic pathway in the ATAAD group and a remaining heparin effect in the control group. In the ATAAD group, FIBTEM is normalized and equal to the control group. These findings are in line with similar work by Liu et al. and Guan et al. who did serial TEG analysis on patients with ATAAD ${ }^{21,22}$. Data in both studies demonstrated that ATAAD initiates a consumption coagulopathy, and surgery affects fibrinogen and clotting factors more than platelets. However, TEG is not able to detect differences between intrinsic and extrinsic pathways. The studies lacked either a control group or did not provide differences between samples with heparinase.

Postoperatively, the patient was hypercoagulable in both groups, primarily in terms of FIBTEM, which may be explained by an increase in fibrinogen levels caused by inflammation ${ }^{23}$. ROTEM at day 4-5 $\left(\mathrm{T}_{5}\right)$ showed normal CT in INTEM and a prolonged CT in EXTEM in both groups. The MCF was elevated in FIBTEM and EXTEM. The increase is likely driven by high fibrinogen levels. The prolonged CT in EXTEM indicates a higher threshold for extrinsic pathway activation, likely induced by increased activity by inhibiting factors such as antithrombin, protein $\mathrm{C}$, and protein $\mathrm{S}$. This is supported by the normal PT-INR and increased antithrombin at day five as shown previously ${ }^{17}$.

Point-of-care testing with either ROTEM or tromboelastography (TEG) has been proven to reduce the need for red blood cell transfusion and reduce bleeding in cardiac surgery ${ }^{10}$. The clotting time (CT) of INTEM and EXTEM test the same pathways as activated partial thromboplastin time (APTT) and prothrombin time (PT-INR), respectively. Maximum clot firmness (MCF) in both INTEM and EXTEM is an estimate of platelet number and fibrinogen levels while MCF in FIBTEM is primarily a measurement of fibrinogen 
levels ${ }^{11}$. One of the main benefits of ROTEM are its fast results compared to compared to routine plasmabased laboratory coagulation tests (RLT) which enables serial testing of the coagulation and allows for faster response on changes in the coagulation during and after surgery, and our results generated by ROTEM are in line with previous reports using RLT ${ }^{17,}{ }^{24}$. However, one main difference between ROTEM and RLT is that RLT has well-established quality assurance programs with imprecision results, coefficient of variation $(\mathrm{CV} \%)<5 \%$, which is more difficult to achieve for a whole-blood system like ROTEM, reflected in the reference values that are wider in ROTEM counterparts. Another benefit of RLT is that ROTEM is harder to interpret and in the setting of complex coagulopathy, the use of both RLT and ROTEM provides a more nuanced picture ${ }^{25}$.

To be able to use ROTEM as a substitute for RLT in a transfusion protocol it needs to adequately identify differences in coagulation seen in RLT. When comparing our ROTEM data with RLT in the same cohort, we find that ROTEM does not detect as many pathologies as RLT does. ROTEM identified all cases with low levels of fibrinogen, a finding supported by previous studies ${ }^{13,} 26$. However, low levels of clotting factors and platelets seem to be underdiagnosed by ROTEM. In this study, ROTEM suggests that $35 \%$ of the patients require PCC or FFP, but RLT indicates that all patients have decreased levels of coagulation factors. This is also demonstrated by Rugeri et al. ${ }^{26}$ who showed poor correlation between CT and APTT/PT-INR. Platelet levels were also underdiagnosed by ROTEM, where only one out of seven thrombocytopenic patients were detected compared to RLT.

Our ROTEM-guided transfusion protocol was introduced in 2015. It follows a similar structure to previously published protocols ${ }^{9,10,12}$. The adherence to protocol in this study, however, was not always optimal. When analyzing the transfusions, all patients in the ATAAD group received platelets, fibrinogen, and PCC and/or FFP. Compared to the transfusion protocol, all patients in the ATAAD group met the criteria for PCC/FFP substitution, $>80 \%$ for fibrinogen, but only $50 \%$ met the criteria for platelets and $\mathrm{RBC}$ at $\mathrm{T}_{2}$. This could be interpreted in different ways: either pre-emptive transfusions were used to prevent coagulopathy or the protocol was bypassed due to clinical coagulopathy not detected by the protocol. As mentioned earlier, ROTEM failed to identify the patients with low levels of coagulation factors or platelets indicating that the ROTEM-guided algorithm used in routine surgery may not be directly translated to ATAAD or other complex surgery. Also, our ROTEM does not contain platelet function analysis, which may explain why the algorithm was not followed for platelet transfusion.

Although ROTEM has been proven to reduce transfusions in cardiac surgery, some studies show no predictive value of ROTEM ${ }^{11,14-16,27}$. One potential reason might be its imprecision. In elective surgery, a preoperative test enables the patient to act as its own control, and changes in ROTEM could better be interpreted in that context. However, in the acute setting of surgery for ATAAD, the patients' preoperative values are impaired, and preoperative levels have a limited value. This is supported by several studies of ROTEM and TEG showing that comparing pre- and post CPB levels is better at predicting bleeding rather than a post CPB ROTEM/TEG analyzed with predetermined cut-off values ${ }^{11,14-16,27 .}$

There are several limitations to this study. First, the sample size is not large leaving room for type II errors. However, including more subjects would require a longer inclusion period, and smaller differences found in a larger cohort may have questionable clinical value. The use of a control group undergoing aortic surgery is of benefit, but a better-matched surgical procedure with deep hypothermic circulatory arrest could possibly have given even more insights. Since adherence to the transfusion protocol was impaired, the true effect of a ROTEM-guided protocol could not be evaluated.

\section{Conclusion}

ATAAD induces an activation of the coagulation system that affects all three subsystems: the extrinsic, the intrinsic and the common pathway. ROTEM does not adequately catch the full coagulopathy in ATAAD. A transfusion protocol in ATAAD should be specifically created to target this complex coagulopathic state and ROTEM does not negate the need for routine laboratory tests.

Table 1 


\begin{tabular}{|c|c|c|c|}
\hline & $\operatorname{ATAAD}(\mathrm{n}=23)$ & Control $(n=20)$ & p-value \\
\hline$\overline{\text { Age (years) }}$ & $64.6 \pm 11.1$ & $59.2 \pm 14.2$ & 0.17 \\
\hline Sex (Female) & $7(30 \%)$ & $3(15 \%)$ & 0.29 \\
\hline Hypertension & $14(61 \%)$ & $11(55 \%)$ & 0.70 \\
\hline Marfan & $0(0 \%)$ & $2(10 \%)$ & 0.21 \\
\hline $\begin{array}{l}\text { Other connective } \\
\text { disorder }\end{array}$ & $0(\%)$ & $0(0 \%)$ & 1 \\
\hline Bicuspid valve & $0(0 \%)$ & $6(32 \%)$ & $<0.01$ \\
\hline Aortic aneurysm & $6(26 \%)$ & $20(100 \%)$ & $<0.001$ \\
\hline Diabetes & $0(0 \%)$ & $1(5 \%)$ & 0.47 \\
\hline Smoking history & $7(30 \%)$ & $4(27 \%)$ & 1 \\
\hline Chronic kidney disease & $1(4 \%)$ & $0(0 \%)$ & 1 \\
\hline COPD & $0(0 \%)$ & $1(5 \%)$ & 0.47 \\
\hline Stroke & $1(4 \%)$ & $0(0 \%)$ & 1 \\
\hline Aspirin & $3(13 \%)$ & $2(10 \%)$ & 1 \\
\hline Hyperlipidemia & $1(4 \%)$ & $6(30 \%)$ & 0.04 \\
\hline $\begin{array}{l}\text { Proximal surgical } \\
\text { technique }\end{array}$ & & & $<0.001$ \\
\hline - Supracoronary graft & $21(91 \%)$ & $3(6 \%)$ & \\
\hline $\begin{array}{l}\text { - Supracoronary graft } \\
+ \text { AVR }\end{array}$ & $1(4 \%)$ & $1(5 \%)$ & \\
\hline - Root replacement & $1(4 \%)$ & $16(80 \%)$ & \\
\hline $\begin{array}{l}\text { - Valve sparing root } \\
\text { replacement }\end{array}$ & $0(0 \%)$ & $8(40 \%)$ & \\
\hline - Bentall & $1(4 \%)$ & $8(40 \%)$ & \\
\hline $\begin{array}{l}\text { Distal surgical } \\
\text { technique }\end{array}$ & & & 0.04 \\
\hline - Supracoronary graft & $21(91 \%)$ & $16(80 \%)$ & \\
\hline - Hemiarch & $0(0 \%)$ & $4(20 \%)$ & \\
\hline - Arch & $2(9 \%)$ & $0(0 \%)$ & \\
\hline Arterial cannulation & & & $<0.001$ \\
\hline - Femoral & $18(78 \%)$ & $0(0 \%)$ & \\
\hline - Arch & $1(4 \%)$ & $17(85 \%)$ & \\
\hline - Other & $4(17 \%)$ & $3(15 \%)$ & \\
\hline Symptom duration (h) & $7.0(3.9)$ & & \\
\hline Operation time (min) & $298 \pm 72$ & $322 \pm 79$ & 0.10 \\
\hline CPB time (min) & $160 \pm 36$ & $208 \pm 60$ & $<0.01$ \\
\hline $\mathrm{Cx}$ time (min) & $75 \pm 31$ & $139 \pm 44$ & $<0.001$ \\
\hline HCA time (min) & $21.6 \pm 10.5$ & $3.6 \pm 8.2$ & $<0.001$ \\
\hline SCP modality & & & $<0.001$ \\
\hline - Circulatory arrest & $14(61 \%)$ & $0(0 \%)$ & \\
\hline - SABP & $9(39 \%)$ & $4(20 \%)$ & \\
\hline SCP time & & 0 & $<0.001$ \\
\hline Lowest core temp $\left({ }^{\circ} \mathrm{C}\right)$ & $20.0 \pm 2.8$ & $30.5 \pm 3.1$ & $<0.001$ \\
\hline $\begin{array}{l}\text { Circulatory arrest time } \\
(\min )\end{array}$ & $17.9 \pm 5.8$ & 0 & $<0.01$ \\
\hline
\end{tabular}




\begin{tabular}{|c|c|c|c|}
\hline & $\operatorname{ATAAD}(\mathrm{n}=23)$ & Control $(\mathrm{n}=20)$ & $\mathrm{p}$-value \\
\hline $\begin{array}{l}\text { Values are expressed as } \\
\text { numbers (\%) or mean } \\
\pm \text { SD. ATAAD, acute } \\
\text { aortic dissection; } \\
\text { COPD, chronic } \\
\text { obstructive pulmonary } \\
\text { disease; AVR, aortic } \\
\text { valve replacement; } \\
\text { CPB, cardiopulmonary } \\
\text { bypass; Cx, crossclamp; } \\
\text { HCA, hypothermic } \\
\text { circulatory arrest; SCP, } \\
\text { selective cerebral } \\
\text { perfusion; SABP, } \\
\text { selective antegrade } \\
\text { brain perfusion. }\end{array}$ & $\begin{array}{l}\text { Values are expressed as } \\
\text { numbers (\%) or mean } \\
\pm \text { SD. ATAAD, acute } \\
\text { aortic dissection; } \\
\text { COPD, chronic } \\
\text { obstructive pulmonary } \\
\text { disease; AVR, aortic } \\
\text { valve replacement; } \\
\text { CPB, cardiopulmonary } \\
\text { bypass; Cx, crossclamp; } \\
\text { HCA, hypothermic } \\
\text { circulatory arrest; SCP, } \\
\text { selective cerebral } \\
\text { perfusion; SABP, } \\
\text { selective antegrade } \\
\text { brain perfusion. }\end{array}$ & $\begin{array}{l}\text { Values are expressed as } \\
\text { numbers (\%) or mean } \\
\pm \text { SD. ATAAD, acute } \\
\text { aortic dissection; } \\
\text { COPD, chronic } \\
\text { obstructive pulmonary } \\
\text { disease; AVR, aortic } \\
\text { valve replacement; } \\
\text { CPB, cardiopulmonary } \\
\text { bypass; Cx, crossclamp; } \\
\text { HCA, hypothermic } \\
\text { circulatory arrest; SCP, } \\
\text { selective cerebral } \\
\text { perfusion; SABP, } \\
\text { selective antegrade } \\
\text { brain perfusion. }\end{array}$ & $\begin{array}{l}\text { Values are expressed as } \\
\text { numbers (\%) or mean } \\
\pm \text { SD. ATAAD, acute } \\
\text { aortic dissection; } \\
\text { COPD, chronic } \\
\text { obstructive pulmonary } \\
\text { disease; AVR, aortic } \\
\text { valve replacement; } \\
\text { CPB, cardiopulmonary } \\
\text { bypass; Cx, crossclamp; } \\
\text { HCA, hypothermic } \\
\text { circulatory arrest; SCP, } \\
\text { selective cerebral } \\
\text { perfusion; SABP, } \\
\text { selective antegrade } \\
\text { brain perfusion. }\end{array}$ \\
\hline
\end{tabular}

Table 2.

\begin{tabular}{l}
\hline Early mortality \\
Chest output (ml) \\
$-12 \mathrm{~h}$ \\
$-24 \mathrm{~h}$ \\
Transfusions \\
Packed red blood cells $(\mathrm{U})$ \\
Platelets (U) \\
Fresh frozen plasma (U) \\
Fibrinogen (g) \\
Novoseven \\
Antithrombin \\
Tranexamic acid (g) \\
Desmopressin ( $\mu \mathrm{g})$ \\
PCC (IE) \\
Re-exploration for bleeding \\
Postoperative stroke \\
Dialysis (RRT) \\
Prolonged ventilation ( $>48 \mathrm{~h})$ \\
ICU stay (days) \\
Values are expressed as numbers $(\%)$ or mean \pm SD. ATAAD, acute aortic dissection; PCC, prothrombin complex concentra \\
\hline
\end{tabular}

\section{FIGURE LEGENDS}

FIGURE 1. Flow chart of patients with acute aortic syndromes referred to the Department of Cardiothoracic Surgery, Skåne University Hospital, Lund, Sweden, during the inclusion period.

FIGURE 2. Transfusion protocol

FIGURE 3. ROTEM analyses 


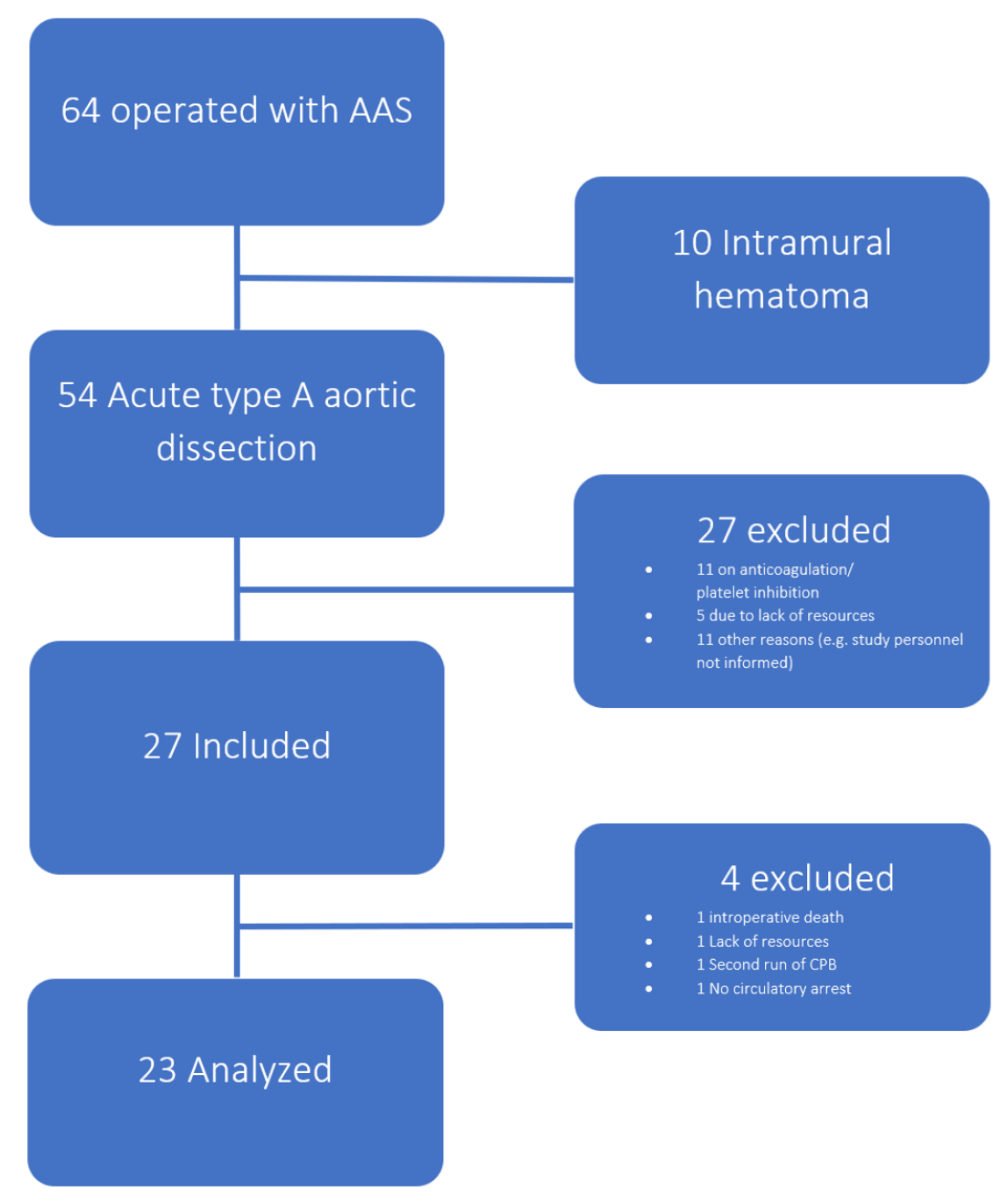

Figure 1.

Figure 2. 


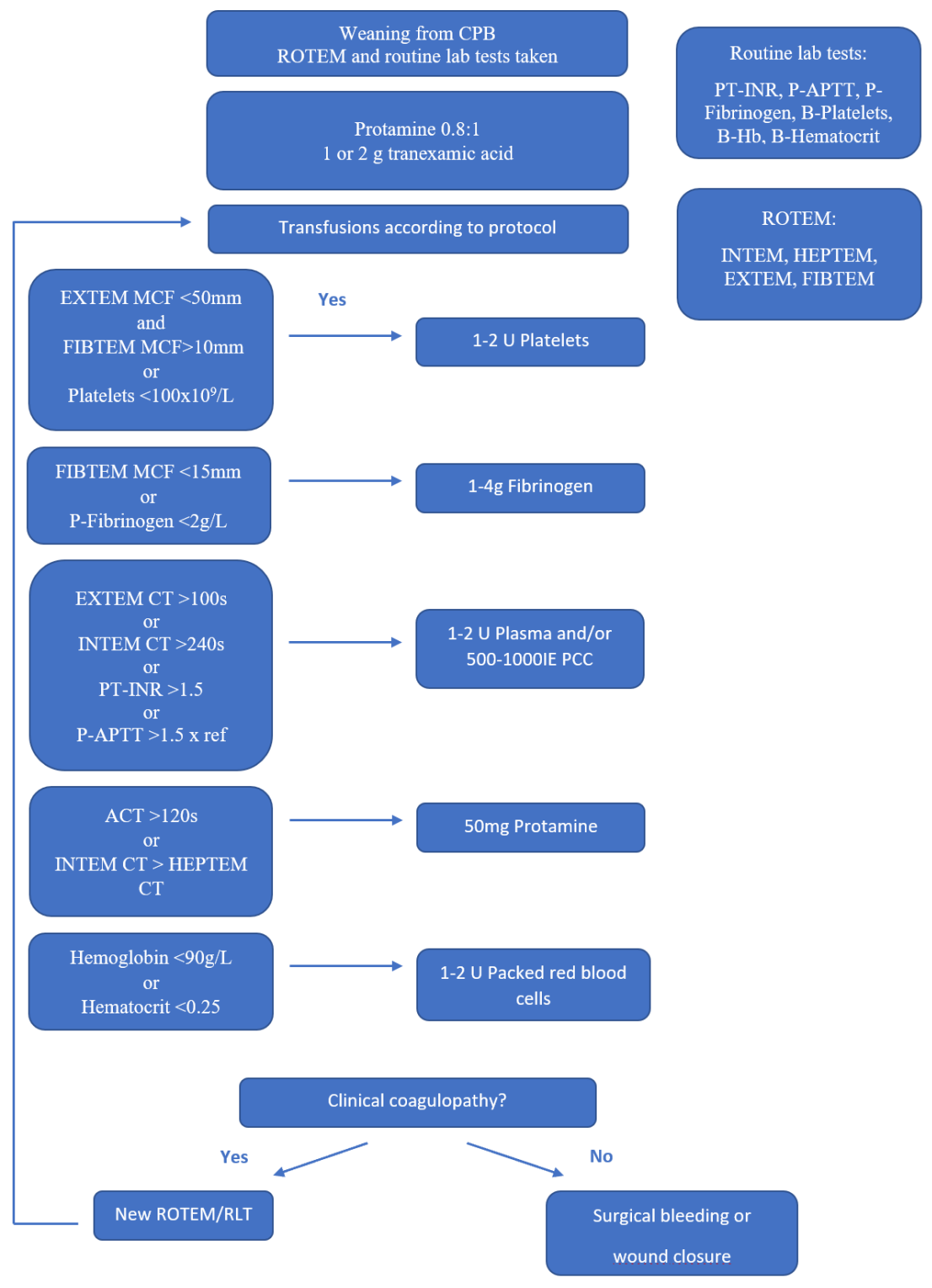

Figure 3 . 

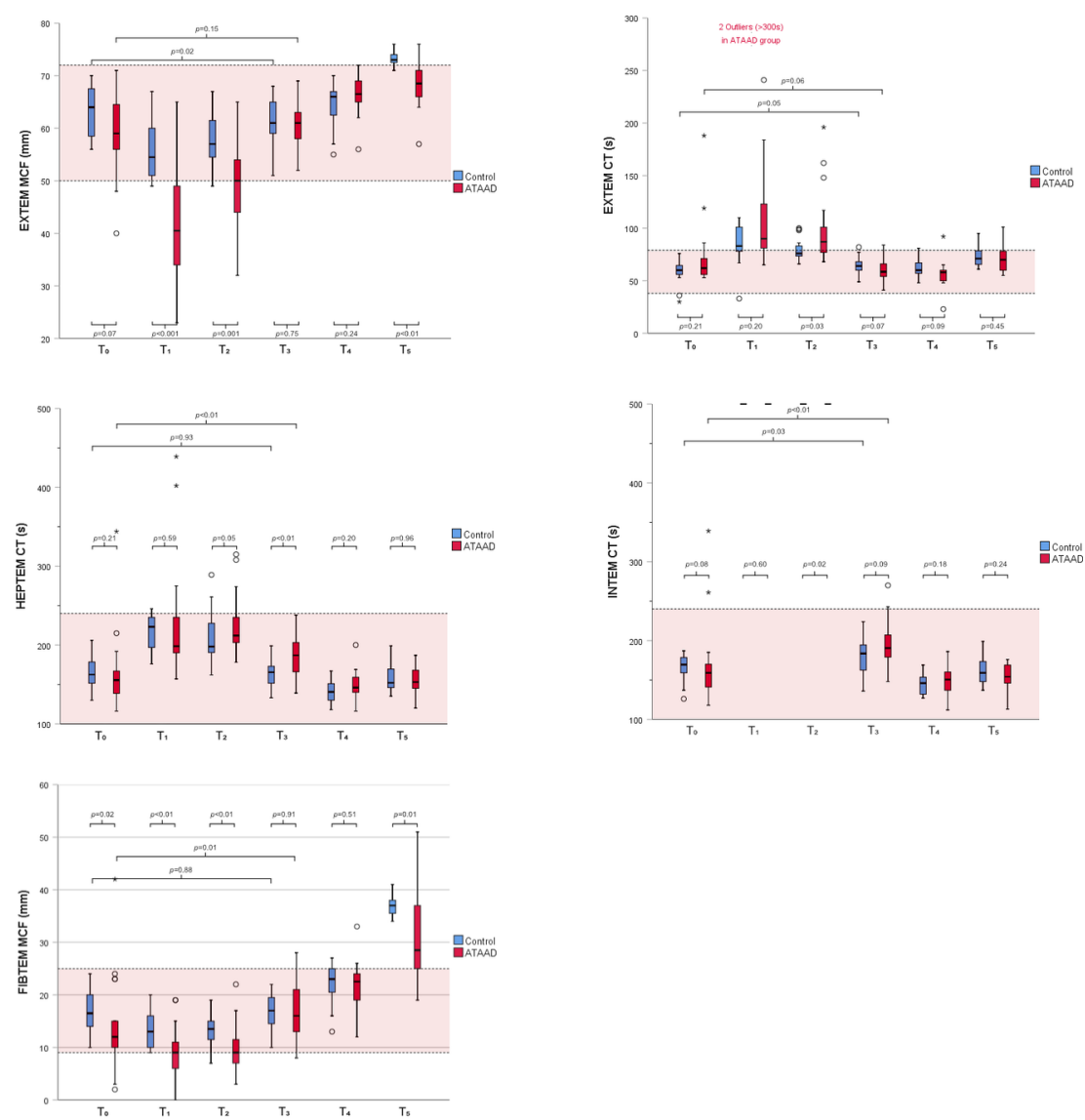

\section{REFERENCES}

1. Czerny M, Schoenhoff F, Etz C, et al. The Impact of Pre-Operative Malperfusion on Outcome in Acute Type A Aortic Dissection: Results From the GERAADA Registry. J Am Coll Cardiol 2015;65(24):2628-2635.

2. Evangelista A, Isselbacher EM, Bossone E, et al. Insights From the International Registry of Acute Aortic Dissection: A 20-Year Experience of Collaborative Clinical Research. Circulation 2018;137(17):1846-1860.

3. Geirsson A, Shioda K, Olsson C, et al. Differential outcomes of open and clamp-on distal anastomosis techniques in acute type A aortic dissection. J Thorac Cardiovasc Surg 2019;157(5):1750-1758.

4. Zindovic I, Sjogren J, Bjursten H, et al. Predictors and impact of massive bleeding in acute type A aortic dissection. Interact Cardiovasc Thorac Surg 2017;24(4):498-505.

5. Hofer J, Fries D, Solomon C, et al. A Snapshot of Coagulopathy After Cardiopulmonary Bypass. Clin Appl Thromb Hemost 2016;22(6):505-511.

6. Koster A, Fischer T, Praus M, et al. Hemostatic activation and inflammatory response during cardiopulmonary bypass: impact of heparin management. Anesthesiology 2002;97(4):837-841.

7. Ranucci M, Baryshnikova E, Ciotti E, et al. Hemodilution on Cardiopulmonary Bypass: Thromboelastography Patterns and Coagulation-Related Outcomes. J Cardiothorac Vasc Anesth 2017;31(5):1588-1594.

8. Chee YE, Liu SE, Irwin MG. Management of bleeding in vascular surgery. Br J Anaesth 2016;117 Suppl 
2:ii85-ii94.

9. Girdauskas E, Kempfert J, Kuntze T, et al. Thromboelastometrically guided transfusion protocol during aortic surgery with circulatory arrest: a prospective, randomized trial. J Thorac Cardiovasc Surg 2010;140(5):1117-1124 e1112.

10. Gorlinger K, Perez-Ferrer A, Dirkmann D, et al. The role of evidence-based algorithms for rotational thromboelastometry-guided bleeding management. Korean J Anesthesiol 2019;72(4):297-322.

11. Tanaka KA, Bolliger D, Vadlamudi R, et al. Rotational thromboelastometry (ROTEM)-based coagulation management in cardiac surgery and major trauma. J Cardiothorac Vasc Anesth 2012;26(6):1083-1093.

12. Weber CF, Gorlinger K, Meininger D, et al. Point-of-care testing: a prospective, randomized clinical trial of efficacy in coagulopathic cardiac surgery patients. Anesthesiology 2012;117(3):531-547.

13. Ogawa S, Szlam F, Chen EP, et al. A comparative evaluation of rotation thromboelastometry and standard coagulation tests in hemodilution-induced coagulation changes after cardiac surgery. Transfusion 2012;52(1):14-22.

14. Davidson SJ, McGrowder D, Roughton M, et al. Can ROTEM thromboelastometry predict postoperative bleeding after cardiac surgery? J Cardiothorac Vasc Anesth 2008;22(5):655-661.

15. Lee GC, Kicza AM, Liu KY, et al. Does rotational thromboelastometry (ROTEM) improve prediction of bleeding after cardiac surgery? Anesth Analg 2012;115(3):499-506.

16. Wasowicz M, McCluskey SA, Wijeysundera DN, et al. The incremental value of thrombelastography for prediction of excessive blood loss after cardiac surgery: an observational study. Anesth Analg 2010;111(2):331-338.

17. Zindovic I, Sjogren J, Bjursten H, et al. The Coagulopathy of Acute Type A Aortic Dissection: A Prospective, Observational Study. J Cardiothorac Vasc Anesth 2019;33(10):2746-2754.

18. Daily PO, Trueblood HW, Stinson EB, et al. Management of acute aortic dissections. Ann Thorac Surg 1970;10(3):237-247.

19. Debakey ME, Henly WS, Cooley DA, et al. Surgical Management of Dissecting Aneurysms of the Aorta. J Thorac Cardiovasc Surg 1965;49:130-149.

20. Lang T, Bauters A, Braun SL, et al. Multi-centre investigation on reference ranges for ROTEM thromboelastometry. Blood Coagul Fibrinolysis 2005;16(4):301-310.

21. Guan X, Li J, Gong M, et al. The hemostatic disturbance in patients with acute aortic dissection: A prospective observational study. Medicine (Baltimore) 2016;95(36):e4710.

22. Liu Y, Han L, Li J, et al. Consumption coagulopathy in acute aortic dissection: principles of management. J Cardiothorac Surg 2017;12(1):50.

23. Castell JV, Gomez-Lechon MJ, David M, et al. Acute-phase response of human hepatocytes: regulation of acute-phase protein synthesis by interleukin-6. Hepatology 1990;12(5):1179-1186.

24. Guan XL, Wang XL, Liu YY, et al. Changes in the Hemostatic System of Patients With Acute Aortic Dissection Undergoing Aortic Arch Surgery. Ann Thorac Surg 2016;101(3):945-951.

25. Sahli SD, Rossler J, Tscholl DW, et al. Point-of-Care Diagnostics in Coagulation Management. Sensors (Basel) 2020;20(15).

26. Rugeri L, Levrat A, David JS, et al. Diagnosis of early coagulation abnormalities in trauma patients by rotation thrombelastography. J Thromb Haemost 2007;5(2):289-295. 
27. Cherng YG, Chao A, Shih RL, et al. Preoperative evaluation and postoperative prediction of hemostatic function with thromboelastography in patients undergoing redo cardiac surgery. Acta Anaesthesiol Sin 1998;36(4):179-186. 